\title{
One-Mediator Model of Exposure Effects Is Still Viable
}

\author{
Michael H. Birnbaum and Barbara A. Mellers \\ University of Illinois at Urbana-Champaign
}

\begin{abstract}
Birnbaum and Mellers criticized the use of partial correlation and multiple regression by Moreland and Zajonc to argue for two independent effects of stimulus exposure on liking. The null hypothesis that one variable mediates the effect of the independent variable on the dependent variables was not tested by their analyses. In response, Moreland and Zajonc reanalyzed their data, using structural equations analysis, and replied that there is evidence to support their previous conclusions. However, the present article shows that the small residuals from the one-mediator model may be due to shared nonlinearity (correlated errors) in three of the dependent variables. This simpler interpretation achieves a better fit to the Moreland and Zajonc data than the two-mediator model they advocated. Since the null hypothesis of one mediator is still viable, the burden of proof rests on those who contend that there is more to the exposure effect than stimulus recognition.
\end{abstract}

Moreland and Zajonc (1977) replicated the exposure effect, that is, the finding that stimuli that are presented with greater frequency will be rated more favorably. They also asked their subjects to rate their familiarity with the stimuli, and they found a significant partial correlation between liking and exposure frequency when rated familiarity was partialed out. This partial correlation (and related regression analyses) led them to conclude that there is an additional, "independent" effect of exposure frequency on liking that is not mediated by stimulus recognition.

Birnbaum and Mellers (1979) questioned the use of partial correlation and regression in this argument. They pointed out that the null hypothesis that a single variable (e.g., recognition) mediates the effect of the independent variable on both dependent variables predicts that both partials should have the same sign as the original correlations. Only if the dependent variable measuring recognition is assumed to be perfectly correlated with the mediator does the regression analy-

Requests for reprints should be sent to Michael H. Birnbaum, Department of Psychology, University of Illinois, Champaign, Illinois 61820 . sis test the null hypothesis. Otherwise, lack of perfect validity of the dependent variable vitiates the analysis.

Birnbaum and Mellers (1979) presented path models both for the null hypothesis of one mediator and for the alternative hypothesis that a second mediator is required. Implications of the models and methods to distinguish them were described. These methods were applied to the three major variables in Moreland and Zajonc (1977) with the result that no evidence was found to require the hypothesis of two mediators over the simpler hypothesis of one.

In response to these arguments, Moreland and Zajonc (1979) reanalyzed their data, using structural equations analysis. They argued that when all five variables are considered, a case can be made for the existence of an extra path from stimulus frequency to liking, in addition to the path via subjective recognition. However, the present article will show that the residuals from the one-mediator model, fit to all five variables, are small in magnitude and are not of the form anticipated by the Moreland and Zajonc model. Instead, they can be explained by the simpler hypothesis that some of the dependent variables are nonlinearly related to the single mediator. 


\section{One or Two Mediators?}

The diagram in Figure 1 is an extension of Birnbaum and Mellers (1979, Expression 9) for all five variables reported by Moreland and Zajonc (1977).

The diagram indicates that the independent variable, frequency of stimulus exposure, affects a mediator, "subjective recognition," which in turn influences the four dependent variables, rated affect (liking), rated familiarity, rated recognition confidence, and recognition accuracy. Moreland and Zajonc (1977, 1979 ) argue that there is an additional, independent effect of stimulus exposure on liking, presumably via a second mediator, labeled "subjective affect" in Figure 1.

The one-mediator model is a special case of Figure 1 with $b=0$. The model favored by Moreland and Zajonc (1979, Figure 1), although written in more complex form, is also a special case of Figure 1, with $c_{1}=c_{2}=$ $c_{3}=0$.

\section{One-Mediator Model}

Table 1 shows the residual correlations that remain after fitting a linear one-mediator model ( $b=0, c_{1}=c_{2}=c_{3}=0$ ) to the correlations for both experiments of Moreland and Zajonc (1977; see Table 1 of Birnbaum \& Mellers, 1979). Models were fit to maximum likelihood criterion by means of ACOVS and LISREL III (Jöreskog, 1974; Jöreskog, Gruvaeus, \& van Thillo, 1970; Jöreskog \& Sörbom, 1976). In general, the residual correlations in Table 1 are small in magnitude. In particular, the largest deviation does not occur for the residual correlation between liking (affect) and frequency (only .03 and .06 for Experiments 1 and 2), which might be expected to be large if there is an extra causal path from frequency to liking. Instead, the largest residual for both experiments is for the correlation between recognition accuracy and recognition confidence (.08 and .18$)$.

One can reasonably ask if the residual correlations in Table 1, which (for Experiment 2) are based on 10 ratings from each of 40 subjects, are of sufficient magnitude to reject the one-mediator model. Moreland and Zajonc (1979) contend that the lack of fit for this

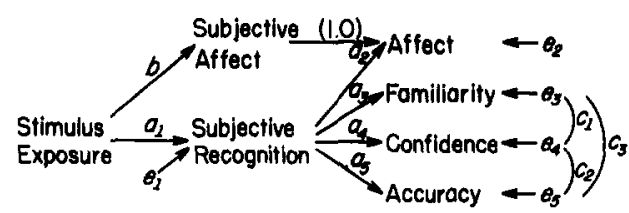

Figure 1. Path diagram representing theories of stimulus exposure. (The coefficient $a_{1}$ represents the correlation between stimulus exposure and a mediator, labeled "subjective recognition," which in turn is correlated with the four dependent variables with coefficients of $a_{2}, a_{3}, a_{1}$, and $a_{n}$. The errors, $e_{1}$ through $e_{5}$ represent residuals, analogous to uniquenesses in factor analysis. The coefficient $b$ represents the effect of stimulus exposure on liking apart from its effect via the first mediator. The correlations $c_{1}, c_{3}$, and $c_{n}$ allow for correlated errors, which could be produced by shared nonlinearity of the dependent variables measuring recognition. The issue at hand is whether the data of Moreland and Zajonc (1977) provide sound evidence to reject the null hypothesis that $b=0$.)

model is evidence of a second mediator. It will be shown below that this pattern of small residual correlations can be better fit by a one-mediator model, which allows the errors in the recognition measures to be correlated (i.e., the $c$ s in Figure 1 are not fixed to zero). Shared nonlinearity of the recognition measures would predict correlated errors.

\section{Nonlinearity in Moreland and Zajonc Data}

Figure $2 \mathrm{~A}$ plots the means of the four dependent variables as a function of the independent variable, log stimulus frequency, as reported in Table 3 of Moreland and Zajonc (1977). The ordinate has been recalibrated linearly so that all four dependent variables can be shown simultaneously. The one-mediator model and the two-mediator model both imply that the curves should all be linear. Should two variables share common nonlinearity, the residual correlation in Table 1 is expected to be positive. All three recognition measures appear to be nonlinearly related to frequency of stimulus exposure. Furthermore, recognition confidence and recognition accuracy appear to share a common cubic trend in relation to the other curves. A similar pattern was evident for Experiment 1. Thus, the two variables that show the largest residual from the one-mediator model (Table 
Table 1

Residuals From One-Mediator Linear Model

\begin{tabular}{lccccc}
\hline & Frequency & Liking & Familiarity & Confidence & Accuracy \\
\hline Frequency & & .03 & .01 & .02 & -.07 \\
Affect & .06 & & -.01 & .02 & -.06 \\
Familiarity & -.03 & -.02 & & -.06 & .04 \\
Confidence & -.01 & -.06 & -.02 & .08 \\
Accuracy & -.05 & -.11 & .13 & .18 & \\
\hline
\end{tabular}

Note. Each entry is the residual correlation after fitting a one-mediator model to the correlations obtained by Moreland and Zajonc (1977). Values above the diagonal are for Experiment 1 ; values below the diagonal are for Experiment 2. Liking and affect are corresponding measures for Experiments 1 and 2, respectively. The question under consideration is whether correlations of this pattern and magnitude, based on 10 judgments from each of 40 subjects (Experiment 2), warrant rejection of the one-mediator model. Note that the largest residuals involve accuracy, which is nonlinearly related to frequency (Figure 2).

1) are the two that are most nonlinearly related to the others (Figure 2A).

\section{Nonlinearity Can Produce Correlated Residuals}

To illustrate how shared nonlinearity can produce deviations from a one-mediator model, a hypothetical data set was constructed from the following one-mediator model:

$$
\begin{aligned}
& F=X+e_{1} ; \\
& Y_{1}=F+e_{2} ; \\
& Y_{2}=F+.33 F^{2}+e_{3} ; \\
& Y_{3}=F+.33 F^{2}+e_{4} ; \\
& Y_{4}=F+.33 F^{2}+e_{5} ;
\end{aligned}
$$

where $X$ is the manipulated independent variable (analogous to stimulus exposure in Figure 1); $Y_{1}$ through $Y_{4}$ are the measured, dependent variables; $F$ is the mediating factor (analogous to subjective recognition); the addition of $.33 F^{2}$ produces nonlinear relationships between $Y_{2}$ through $Y_{4}$ and $F$; and $e_{1}$ through $e_{5}$ are mutually uncorrelated error terms. The value .33 for the coefficient of $F^{2}$ was chosen so that the degree of nonlinearity would be roughly comparable to that in the data of Moreland and Zajonc (1977), as in Figure 2A. To generate the 160 hypothetical cases, 5 values of $X,(-2,-1,0,1,2)$ were factorially combined with 2 values of $e_{1}(-1$, 1 ) to produce 10 values of $F$. These values of $F$ were factorially combined with two levels of each error $\left(e_{2}=-1.1,1.1 ; e_{3}=-4,4\right.$; $\left.e_{4}=-3,3 ; e_{5}=-2,2\right)$ to generate the val- ues of the dependent variables, $Y_{1}, Y_{2}, Y_{3}$, and $Y_{4}$, according to Equation 1. The mean values for $Y$ for each level of $X$ are shown in Figure 2B, plotted for comparison with Figure 2A. For the hypothetical example, variables $Y_{2}, Y_{3}$, and $Y_{4}$ share a common nonlinear (quadratic) relationship with the other two variables. If the data were generated without the $F^{2}$ terms, the curves would be linear; therefore, the linear one-mediator model (with $b=c_{1}=c_{2}=c_{3}$ fixed to zero in Figure 1) would fit perfectly.

The correlation matrix, generated from the

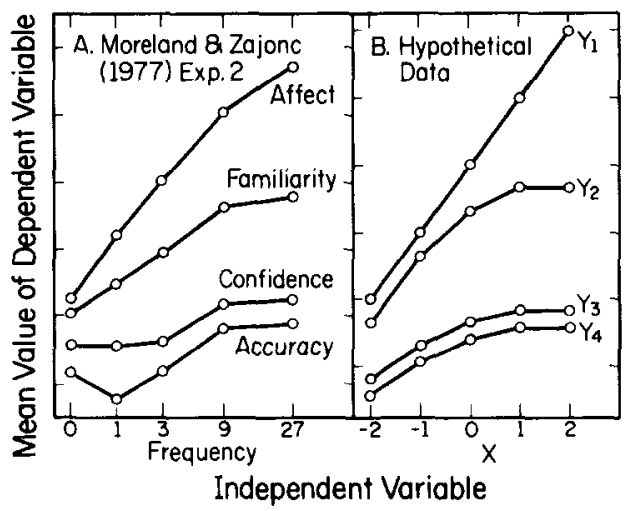

Figure 2. A. Mean values of affect, rated familiarity, recognition confidence, and recognition accuracy as a function of exposure frequency $[\log (f+1)]$. (From Moreland \& Zajonc, 1977, Table 3. Ordinate separately calibrated for each variable.) B. Mean values of dependent variables, $Y_{1}, Y_{2}, Y_{\mathrm{a}}$, and $Y_{4}$, as a function of independent variable. (Hypothetical data were generated from a one-mediator model with $Y_{2}, Y_{\mathrm{a}}$, and $Y_{4}$ nonlinearly related to the mediating variable, as shown in Equation 1.) 
one-mediator model of Equation 1 (with some dependent variables nonlinearly related to the mediator), is shown in the upper portion of Table 2. The correlation matrix was fit by means of LISREL to a linear one-mediator model $\left(b, c_{1}, c_{2}\right.$, and $c_{3}$ in Figure 1 were fixed to zero). The residual correlations are shown below the diagonal in Table 2. Note that the largest discrepancies in Table 2 are among variables $Y_{2}, Y_{3}$, and $Y_{4}$, which have nonlinearity (i.e., quadratic term, $F^{2}$, in Equation 1) in common. The residual correlation between $X$ and $Y_{1}$ is also positive, since the best-fit single factor falls between $F$ and $F^{2}$. This pattern of residuals is similar to that in Table 1.

A one-mediator model can be fit to the hypothetical data perfectly if the residuals for $Y_{2}, Y_{3}$, and $Y_{4}$ are allowed to be correlated. These correlations represent the shared nonlinearity of these variables. The model in Figure 1 thus fits the hypothetical data perfectly with $b$ set to zero, by allowing correlations $c_{1}, c_{2}$, and $c_{3}$ to be nonzero (the values of $c$ can be further constrained to satisfy Equation 10 of Birnbaum \& Mellers, 1979).

This example illustrates that even when data are constructed from a single-mediator model, if some variables are nonlinearly related, deviations from the model can appear unless the model allows correlations among the residuals for nonlinear variables.

\section{Correlated Residual Model Fits Moreland and Zajonc Data}

The model that fits the hypothetical data (Figure 2B) also achieves a good fit to the Moreland and Zajonc (1977) data (Figure $2 \mathrm{~A})$. It is a special case of a one-mediator model with nonzero correlations among the errors in the recognition variables. The correlations among the residuals $\left(c_{1}, c_{2}\right.$, and $\left.c_{3}\right)$ may be given a response-bias, or "method," interpretation, since this model does not postulate any additional paths from stimulus frequency to any of the variables, besides the one via the recognition mediator ( $b$ is set to zero). The residual correlations from this one mediator model are quite small, the largest deviation being .04 for both experiments. The residual correlations between exposure fre-
Table 2

Hypothetical Example for One-Mediator Nonlinear Model

\begin{tabular}{cccccc}
\hline & $X$ & $Y_{1}$ & $Y_{2}$ & $Y_{3}$ & $Y_{4}$ \\
\hline$X$ & & .69 & .32 & .39 & .49 \\
$Y_{1}$ & .02 & & .32 & .40 & .51 \\
$Y_{2}$ & -.02 & -.02 & & .26 & .37 \\
$Y_{3}$ & -.03 & -.02 & .04 & & .40 \\
$Y_{4}$ & -.02 & -.02 & .06 & .08 & \\
\hline
\end{tabular}

Note. $X=$ independent variable; $Y_{1}$ through $Y_{4}$ $=$ dependent variables. Correlations are above diag. onal; residuals from one-mediator linear model are below diagonal. Note similarity of residuals to those in Table 1.

quency and liking are -.02 and .00 for $\mathrm{Ex}$ periments 1 and 2 , respectively.

Thus, the residual correlations from the linear one-mediator model may be attributed to shared nonlinearity of the recognition measures. This interpretation is consistent with the data shown in Figure 2A, with the pattern of residuals in Table 1 (in which the largest deviations involve recognition accuracy), and with the fact that the one-mediator model with correlated errors provides a good fit to the correlations. In sum, the one-mediator model remains a viable interpretation of the Moreland and Zajonc (1977) data if it is allowed that the recognition measures are nonlinearly related to the other variables.

\section{Two-Mediator Model Not Required}

The two-mediator model favored by Moreland and Zajonc (1979, Figure 1) yields the theoretical correlation matrix shown in the lower triangle of Table 3 . The model is equivalent to Figure 1 of the present article with $c_{1}=c_{2}=c_{3}$ set to zero. Table 3 shows that if $b=0$, each correlation can be represented as the product of correlations with the mediating variable, subjective recognition. However, with $b>0$, the correlations between $Y_{1}$ (liking) and the others increase, especially the correlation between $X$ and $Y_{1}$.

The hypothetical correlations generated from a two-mediator model in the upper portion of Table 3 show that in principle, it is possible to distinguish the two-mediator model from the one-mediator model. Note that the 
Table 3

Theoretical Values for Two-Mediator Model and Hypothetical Example

\begin{tabular}{lccccc}
\hline & $X$ & $Y_{1}$ & $Y_{2}$ & $Y_{3}$ & $Y_{4}$ \\
\hline$X$ & & .75 & .40 & .40 & .40 \\
$Y_{1}$ & $a_{1} a_{2}+b$ & & .60 & .60 & .60 \\
$Y_{2}$ & $a_{1} a_{3}$ & $a_{2} a_{3}+b a_{1} a_{3}$ & & .64 & .64 \\
$Y_{3}$ & $a_{1} a_{4}$ & $a_{2} a_{4}+b a_{1} a_{4}$ & $a_{3} a_{4}$ & & .64 \\
$Y_{4}$ & $a_{1} a_{5}$ & $a_{2} a_{5}+b a_{1} a_{5}$ & $a_{3} a_{5}$ & $a_{4} a_{5}$ & \\
\hline
\end{tabular}

Note. $X=$ independent variable; $Y_{1}$ through $Y_{4}$ $=$ dependent variables. Theoretical values are below diagonal. Hypothetical values above diagonal are based on $a_{1}=a_{2}=.5, a_{3}=a_{4}=a_{5}=.8$, and $b=.5$.

hypothetical correlations of frequency, liking, and familiarity violate Equation 10 of Birnbaum and Mellers (1979), since $.75 / .40=$ 1.88 , which exceeds the limit set by $1 / .60=$ $1.67 .^{1}$

Table 4 shows parameter estimates and an index of fit for the various models under consideration, applied to data for Experiment 2 of Moreland and Zajonc (1977). To obtain the predicted correlation between two variables, multiply the coefficients along each path and sum these products over all distinct paths connecting the two variables in Figure 1 . For example, for the one-mediator model, the predicted correlation between stimulus exposure and rated affect is $a_{1} a_{2}$, or .61. For the two-mediator model, the predicted value would be $a_{1} a_{2}+b$, or .66 .

The two-mediator model shown in Figure 1 of Moreland and Zajonc (1979) is equivalent to the model in Figure 1, with $c_{1}=c_{2}=$ $c_{3}=0$. Because the models are mathematically identical, the index of fit in Table 4 (" $\chi$ ") is the same as reported by Moreland and Zajonc (1979)..2 The largest residual for the two-mediator model for Experiment 2 is .13 , between recognition confidence and recognition accuracy, a slight improvement over the corresponding value of .18 for the onemediator model. ${ }^{\mathrm{s}}$

The one-mediator model with correlated errors provides a better fit to the data than the two-mediator model, although it does not postulate an additional path from exposure to liking. The largest residual for this model is only .04 for both experiments. The value of $\chi^{2}$ is less than one sixth as large as that for the two-mediator model. In addition, permitting $b$ to be nonzero does not improve the fit, once correlations are allowed among the errors in the recognition measures (see last column of Table 4).

In sum, the analyses provide no compelling reason to reject the null hypothesis that $b$ in Figure 1 is zero. Therefore, the data do not demonstrate the existence of another path from stimulus exposure to liking apart from

1 The numerical example in Table 3 also illustrates that the two-mediator model predicts a larger correlation between $X$ and $Y_{1}$ than would be predicted from the one-mediator model. According to the one-mediator model

$$
\hat{\rho}_{X Y_{1}}=\frac{\left(a_{1} a_{3}\right)\left(a_{2} a_{3}\right)\left(a_{4} a_{5}\right)}{\left(a_{3} a_{4}\right)\left(a_{3} a_{5}\right)}=\frac{\rho X Y_{2} \rho Y_{1} Y_{2} \rho Y_{3} Y_{4}}{\rho Y_{2} Y_{3} \rho Y_{2} Y_{4}}
$$

which reduces to $a_{1} a_{2}$. In contrast, the two-mediator theory with $b>0$ prediots that the observed correlation should exceed the value of $\hat{\rho}_{X Y_{1}}$ from Equation 2. For the values used in the hypothetical example of Table 3, Equation 2 (the one-mediator model) yields $\hat{\rho}_{X Y_{1}}=(.40)(.60)(.64) /(.64)(.64)=$ .375 , which is only half as large as the table (twomediator model) value of $\rho_{X Y_{1}}=.75$. When Equation 2 is applied to the correlations of Moreland and Zajonc (1977), the values of $\hat{\rho}_{X Y_{1}}$ are .48 and .81 for Experiments 1 and 2. The obtained values, which by the foregoing should have been larger than these values of $\hat{\rho}_{X Y_{1}}$ according to the twomediator theory, were only .42 and .66 .

2 The model comparisons presented by Moreland and Zajonc (1979) are based on chi-square values that may have been inflated by the assumption that by partialing out the main effect of subjects, the sample of 40 subjects produced 400 independent observations. However, repeated observations from the same subjects should not be treated as if they were independent. Since the computed chi-square is directly proportional to the assumed number of independent observations in each correlation, the " $\chi$ " values reported should not be used for statistical inferences. The " $\chi$ " values reported here are calculated using the same value $(400)$ for the sample size, only to provide an index that can be compared with the accompanying article.

${ }^{3}$ Moreland and Zajonc (1979) fixed the coefficient of familiarity in both of their analyses even though it was actually estimated from their data (see their Footnote 2). As a consequence of this procedure the correct $d f$ for both models are 1 less than they report. 
Table 4

Estimates of Parameters for Model Comparisons

\begin{tabular}{|c|c|c|c|c|}
\hline \multirow[b]{2}{*}{ Variable } & \multicolumn{4}{|c|}{ Model } \\
\hline & One mediator & Two mediator & $\begin{array}{l}\text { One mediator } \\
\text { correlated errors }\end{array}$ & $\begin{array}{l}\text { Two mediator } \\
\text { correlated errors }\end{array}$ \\
\hline$a_{1}$ & .82 & .69 & .86 & .86 \\
\hline$a_{2}$ & .74 & .26 & .77 & .72 \\
\hline$a_{3}$ & .74 & .83 & .68 & .68 \\
\hline$a_{1}$ & .42 & .44 & .37 & .38 \\
\hline$a_{5}$ & .57 & .66 & .45 & .45 \\
\hline$b$ & $(0)$ & .48 & $(0)$ & .04 \\
\hline$c_{1}$ & $(0)$ & (0) & .04 & .03 \\
\hline$c_{2}$ & (0) & (0) & .25 & .25 \\
\hline$c_{3}$ & $(0)$ & (0) & .25 & .24 \\
\hline$d f$ & 5 & 4 & 2 & 1 \\
\hline " $x^{2 "}$ & 83.58 & 38.99 & 6.22 & 6.22 \\
\hline
\end{tabular}

Note. Based on data of Experiment 2 of Moreland and Zajonc (1977). Each entry represents estimated parameter as described in Figure 1, with restrictions imposed by the models. Values shown in parentheses are fixed. Degrees of freedom $(d f)$ are 10 minus the number of estimated parameters. The values of $e_{i}$ are redundant; for example for the one-mediator model, $e_{i}$ can be computed from $1-a_{i}{ }^{2}$. The " $\chi^{2}$ " is inflated and should not be used for statistical tests, but represents an index of fit that can be compared with values reported in the accompanying article.

an effect that could be mediated by stimulus recognition. ${ }^{4}$

It is always preferable to choose among theories on the basis of qualitative differences in predictions for a variety of experimental manipulations rather than on the basis of small differences in an index of fit. Perhaps evidence for a second mediator would occur if a new variable could be found that could potentially reverse the exposure effect, so that repeated exposure could either increase or decrease liking (while increasing recognition), depending on the value of the new variable. A future study may provide sound evidence that stimulus recognition does not mediate the exposure effect. Until such evidence is presented, however, it is premature to assert that there are two independent effects of stimulus exposure on liking.

\section{Conclusions}

These analyses do not disprove the possible existence of unconscious affect, learning without awareness, or subception. They do not disprove the favored interpretation of Moreland and Zajonc (1979), though the one-mediator model with correlated errors fits better than the two-mediator model without correlated errors (Table 4). However, the analyses do show that the null hypothesis of one mediator remains a viable description of the data of Moreland and Zajonc (1977), who argued that there are two independent effects. Perhaps the null hypothesis should be favored by skeptics and the burden of proof be laid upon those who would refute it.

4 Moreland and Zajonc (1979) argued that if a single mediator explains their (1977) data, the mediator may be named "affeot" rather than "recognition." The name of a mediator is a matter of definition that cannot be refuted by experiment. However, any discussion of the proper label for a single mediator is tangential to the original argument of Moreland and Zajonc (1977, p. 193). In that article they stated that evidence of two independent effects of exposure on liking was required to demonstrate that stimulus recognition is not necessary to the exposure effect.

\section{References}

Birnbaum, M. H., \& Mellers, B. A. Stimulus recognition may mediate exposure effects. Journal of Personality and Social Psychology, 1979, 37, 391394.

Jöreskog, K. G. Analyzing psychological data by structural analysis of covariance matrices. In $\mathrm{D}$. 
H. Krantz, R. C. Atkinson, R. D. Luce, \& P. Suppes (Eds.), Contemporary developments in mathematical psychology (Vol. 2). San Francisco: Freeman, 1974.

Jöreskog, K. G., Gruvaeus, G. T., \& van Thillo, M. ACOVS-A general computer program for analysis of covariance structures. Research Bulletin 70-15. Princeton, N.J.: Educational Testing Service, 1970.

Jöreskog, K. G., \& Sörbom, D. LISREL III: Estimation of linear structural equation systems by maxi- mum likelihood methods. Chicago: National Educational Resources, 1976.

Moreland, R. L., \& Zajonc, R. B. Is stimulus recognition a necessary condition for the occurrence of exposure effects? Journal of Personality and Social Psychology, 1977, 35, 191-199.

Moreland, R. L., \& Zajonc, R. B. Exposure effects may not depend on stimulus recognition. Journal of Personality and Social Psychology, 1979, 37, 1085-1089.

Received December 27, 1978

\section{Ad Hoc Reviewers}

The Editor acknowledges with thanks the advice and consultation provided by the following persons, who served recently as ad hoc reviewers of manuscripts. (The number of manuscripts reviewed is in parentheses, if more than one.)

Robert Abelson
John R. Anderson
Norman Anderson
Robert S. Baron
Sandra L. Bem
Michael Birnbaum (4)
Marilyn Brewer
Philip Brickman (2)
Ross W. Buck (2)
Lynn E. Bush
Allan Buss (2)
John Carroll (2)
David W. Chan
George Cicala
Victor Cline
Harris Cooper
James H. Davis
Kay Deaux
Ebbe Ebbesen
Susan Eisen (2)

\author{
Robert Folger \\ Irene Frieze \\ David Funder \\ G. Gerbner \\ Rick Gibbons (2) \\ Wallace Hall \\ Richard J. Harris (3) \\ Elaine Hatfield \\ Chester Insko \\ Carroll Izard \\ Robert L. Kahn \\ David Kenny \\ Vladimir Konečni (2) \\ Gerald Koocher \\ Robert Kraut \\ Arie Kruglanski \\ Michael Kuhlman \\ Lawrence Littig \\ Guy E. Lometti \\ Jacqueline Macaulay
}

\author{
Neil Malamuth \\ Melvin Manis (2) \\ Richard E. Petty (2) \\ Dean Pruitt \\ Dennis Regan (2) \\ Robert Rosenthal \\ Michael Scheier \\ Brendan Rule \\ Barry Schlenker \\ Harold Sigall \\ Dean K. Simonton \\ Shelley Taylor \\ Harry C. Triandis \\ Gary Wells (3) \\ Shirley Weitz \\ Paul Werner \\ Ladd Wheeler \\ Jerry Wiggins (2) \\ Timothy D. Wilson \\ Robert Zajonc
}

